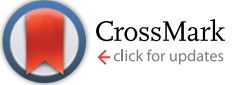

Cite this: RSC Adv., 2017, 7, 361

\title{
Structure effects of amphiphilic and non- amphiphilic quaternary ammonium salts on photodegradation of Alizarin Red-S catalyzed by titanium dioxide $\uparrow$
}

\author{
Paola Anastasio, ${ }^{a}$ Tiziana Del Giacco, ${ }^{\text {*ab }}$ Raimondo Germani, ${ }^{* a b}$ Nicoletta Spreti ${ }^{\mathrm{C}}$ \\ and Matteo Tiecco ${ }^{a}$
}

The role of surfactants such as single- and double-tailed tetraalkylammonium bromide and various nonamphiphilic tetraalkylammonium salts was investigated on the $\mathrm{TiO}_{2}$ photocatalyzed degradation of 3,4dihydroxy-9,10-dioxo-2-anthracenesulfonic acid sodium salt (Alizarin Red-S, ARS) in an air-equilibrated alkaline medium under UV light irradiation. Absorption spectral analysis showed that the photodegradation efficiency of the dye was significantly enhanced by the addition of cationic surfactants. Two interesting findings emerged from this study: ARS was almost completely degraded at surfactant concentrations close to $1 \mathrm{mM}$ (values well above the $\mathrm{cmc}$ in the experimental conditions); moreover, on increasing the surfactant concentration, the photocatalytic reaction became less and less efficient and significantly dependent on the surfactant headgroup size. The presence of a maximum of efficiency depending on the surfactant concentration was due to the combination of catalytic and inhibiting processes. The first one likely depended on the ability of the surfactant to improve the ARS approach to the semiconductor through the formation of cationic bilayers on the $\mathrm{TiO}_{2}$ particles; this effect made the ARS more easily oxidized by $\mathrm{TiO}_{2}$. This catalytic action of the surfactant was opposed by the increase of the micellar aggregate number in the aqueous bulk, which competes with the $\mathrm{TiO}_{2}$ sites in associating to the dye. This was supported by the results obtained with the non-amphiphilic alkylammonium bromide. In this case a higher amount of salt must be added to reach the same maximum efficiency of ARS photooxidation. This is due to the lower capability of neutralization of these salts for both the ARS and the $\mathrm{TiO}_{2}$ surface; the inhibiting effect was not evidenced anymore.

Received 18th October 2016 Accepted 12th December 2016

DOI: $10.1039 / \mathrm{c} 6 \mathrm{ra} 25421 \mathrm{~g}$

www.rsc.org/advances compounds dissolved or dispersed in aqueous media. ${ }^{2-6}$ Among the oxidative photodegradation methods, the use of titanium dioxide has found wide use, because of its photostability, non toxicity, cheapness and strong oxidizing power. Heterogeneous photocatalysis has proved to be an efficient destructive technology, leading to the complete mineralization of most organic pollutants. ${ }^{7-9}$ During the past decade successful improvements in $\mathrm{TiO}_{2}$ photoactivity have been made on the oxidation process: nanostructurated composite materials, as noble metal nanoparticles (Ag, $\mathrm{Au}$ and $\mathrm{Cu}$ NPs), increase the process efficiency when immobilized on semiconductor surface; doped-TiO ${ }_{2}$ with metallic and not metallic species also showed interesting results in this field. ${ }^{\mathbf{1 0}}$ Surfactants play a key-role in this topic, ${ }^{\mathbf{1 1}}$ generally favoring the adsorption of the dye onto the semiconductor surface due to their unique chemical properties.

Previous works reported that $\mathrm{TiO}_{2}$ is a good photocatalyst for ARS (a water-soluble widely used textile dye) and its removal from the aqueous phase. ${ }^{7}$ The efficiency of the dye photodegradation is reported to be influenced by $\mathrm{pH}$, one of the most important factor controlling both the adsorption of dye on the 
semiconductor and the reactivity of the process. In most cases, the photodegradation was performed with UV-illuminated titanium dioxide, but in some works visible radiation by photosensitization was used, certainly finalized to the use of the free and inexhaustible sunlight, of which $40 \%$ is visible light and less than $c a .5 \%$ is UV. Even though there is a basic difference in the primary steps of photodegradation depending on the excitation wavelength (visible or UV irradiation), the photoassisted degradation leads to the dye radical cation formation as an intermediate, which is the main responsible of the degradation of the dye itself. ${ }^{12-15}$

Our long-standing interest in the mechanistic investigation of $\mathrm{TiO}_{2}$ sensitized photooxidation of various organic compound has recently led us to focus our attention on the effect of the surfactant structure ${ }^{\mathbf{1 6 - 1 8}}$ the photodegradation efficiency of ARS catalyzed by $\mathrm{TiO}_{2}$ was studied in air-equilibrated basic aqueous dispersion under UV light irradiation using surfactants differing in their counterions. ${ }^{19}$ A series of surfactants such as cetyltrimethylammonium salts (CTAX, with $\mathrm{X}=\mathrm{Br}^{-}, \mathrm{Cl}^{-}, \mathrm{SO}_{4}{ }^{2-}$, $\mathrm{CH}_{3} \mathrm{SO}_{3}{ }^{-}$, 4- $\left.\mathrm{CH}_{3} \mathrm{C}_{6} \mathrm{H}_{4} \mathrm{SO}_{3}{ }^{-}\right)$were used. From these studies emerged that the photocatalytic efficiency was significantly dependent both on the surfactant concentration and on the nature of the counterion $\mathrm{X}^{-}$.

Changes in the molecular structures of surfactants lead to significant changes in their properties and in the properties of their micellar aggregates. For these reasons the use of synthetic novel amphiphiles represents a step ahead in the chemistry of these molecules, and it can lead to important advantages. ${ }^{20,21}$ The topics where these molecules find an effective role are in fact wide, that is because small chemical modifications of the structures can provoke dramatic changes on their effect. ${ }^{22,23}$ In this work we extended the analysis of the structural effects of tetraalkylammonium bromide surfactants on the $\mathrm{TiO}_{2}$-mediated photodegradation of ARS, considering other surfactants structures, varied in their hydrophobic chains portions and in their headgroup size. Thus we have investigated single-chain surfactants, such as alkylammonium bromide, with different headgroup size (cetyltrimethylammonium, CTA, cetyltriethylammonium, CTEA, cetyltripropylammonium, CTPA, and cetyltributylammonium, CTBA) and variable length tail (dodecyltrimethyl, DTA), and gemini surfactants, with varying tail and two quaternary ammonium headgroups connected by a diethylene oxide spacer (2-[2(dimethylalkylazanium)ethoxy] ethyl-dimethylalkylazanium, Obisalkyl-TA). The comparison between single-chain and gemini systems has given also information on the role played by positive charge number of the surfactant headgroups. All the surfactant structures are shown in Scheme 1. The use of non-amphiphilic ammonium salts in $\mathrm{TiO}_{2}$-catalyzed degradation processes has not received the same attention of amphiphilic salts so far; ${ }^{24-26}$ however this could help the understanding of the role of the headgroup portions of the surfactants in this process. Therefore in this work we have also compared the ability of the various surfactants in assisting the ARS degradation with that of not amphiphilic alkylammonium salts (structures in Scheme 2), such tetraalkylammonium bromide (TRABr), 6-azoniaspiro[5.5]undecane bromide (ASBr), 6,9-diazoniaspiro[5.2.3.2.] hexadecane dibromide (DASBr), butylquinuclidinium bromide (BQBr) and

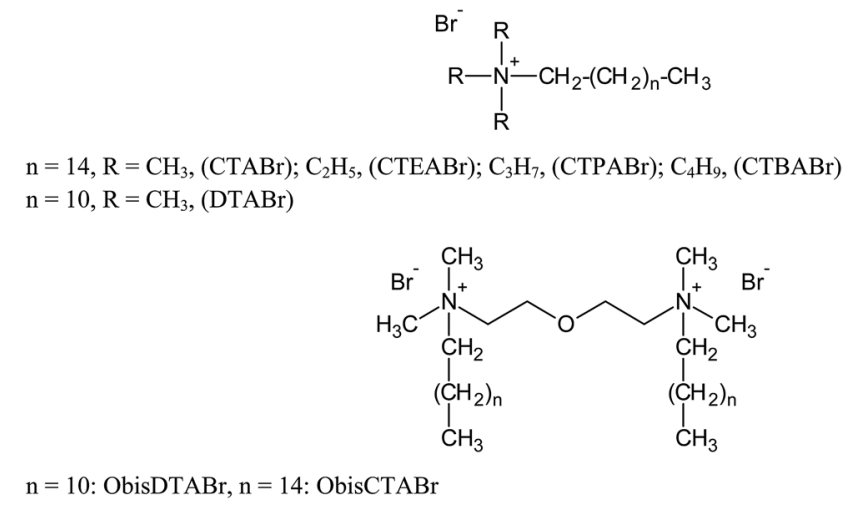

Scheme 1 Cationic surfactants used in this work.<smiles>CC[N+](CC)(CC)CC</smiles>

$\mathrm{n}=0: \mathrm{TMABr}, \mathrm{n}=1: \mathrm{TEABr}, \mathrm{n}=2: \mathrm{TPABr}, \mathrm{n}=3: \mathrm{TBABr}$<smiles>BrC1CCCC[N+]12CCCCC2</smiles>

$\mathrm{ASBr}$

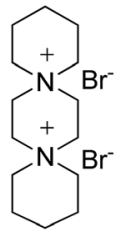

$\mathrm{DASBr}$

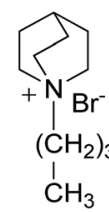

$\mathrm{BQBr}$

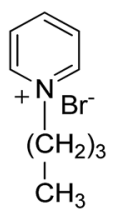

$\mathrm{BPyBr}$
Scheme 2 Quaternary ammonium salts used in this work.

butylpyridinium bromide (BPyBr). This comparison has shown that the hydrophobic degree of the salt strongly influences the reactivity of the oxidation process of the dye.

\section{Experimental}

\section{Materials}

Alizarin Red S (Carlo Erba) and $\mathrm{TiO}_{2}$ (P25, Degussa, 99.5\%, dried in vacuo) were analytical grade reagents. Milli-Q water (18 $\mathrm{M} \Omega$, pH 5.9) was used for all photodegradation experiments. CTABr and DTABr surfactants were purchased from SigmaAldrich and recrystallized from acetone and methanol before use. CTEABr, CTPABr, CTBABr, ObisCTABr and ObisDTABr surfactants were synthesized by quaternization reaction of the correspondent tertiary amines with the appropriate alkyl halide and purified by subsequent crystallizations from acetone or ethyl acetate with different amounts of methanol. ${ }^{27}$ Quaternary ammonium salts TMABr, TEABr, TPABr and TBABr (SigmaAldrich and Carlo Erba, purity $>98 \%$ ) were dried in vacuo over $\mathrm{P}_{2} \mathrm{O}_{5}$ before use. Spiro cyclic quaternary ammonium salts $\mathrm{ASBr}$ and DASBr were synthesized as reported in literature. ${ }^{28} \mathrm{BQBr}$ and $\mathrm{BPyBr}$ were synthesized by quaternization reaction of the 
correspondent tertiary amines with butyl bromide and purified by subsequent crystallizations from acetone/methanol mixture.

\section{Photodegradation experiments}

An identical procedure was followed both with surfactants and with non-amphiphilic salts. The degradation experiments were performed by an Applied Photophysics multilamp apparatus with 12 phosphor-coated fluorescent lamps ( $15 \mathrm{~W}$ each) emitting at $355 \mathrm{~nm}\left(\Delta \lambda_{1 / 2}=20 \mathrm{~nm}\right)$, radiation adsorbed mainly by the semiconductor, as the dye absorbs more weakly at these wavelengths. ${ }^{29}$

$\mathrm{TiO}_{2}$ suspensions were prepared by adding $\mathrm{P} 25 \mathrm{TiO}_{2}$ powder $\left(0.4 \mathrm{~g} \mathrm{l}^{-1}\right)$ to $50 \mathrm{ml}$ of an aqueous solution containing ARS $(0.20$ $\mathrm{mM}$ ) and various concentrations of ammonium salt. The degradations were carried out at $20^{\circ} \mathrm{C}$. The starting $\mathrm{pH}$ of the irradiated suspensions was adjusted to 12 using $\mathrm{NaOH}$. Before irradiation, the suspensions were magnetically stirred in the dark for $30 \mathrm{~min}$ to reach equilibrated adsorption between $\mathrm{TiO}_{2}$, dye and atmospheric oxygen. The irradiated dispersion was kept under constant air-equilibrated and stirred condition.

\section{Analytical procedures}

The degradation process was followed by UV-Vis spectrophotometric analysis. Mixture samples $(2 \mathrm{ml})$ were collected at different irradiation time. To recover quantitatively the dye adsorbed on $\mathrm{TiO}_{2}$, an equal volume of ethanol was added to the sample, together with $0.2 \mathrm{ml}$ of $\mathrm{HCl} 1.2 \mathrm{M}$; in this condition ARS was all protonated. The suspension was then shaken by vortex mixer and centrifuged at $10{ }^{\circ} \mathrm{C}$ for $15 \mathrm{~min}$ at $12000 \mathrm{rpm}$ to remove $\mathrm{TiO}_{2}$. The supernatant $(2 \mathrm{ml})$ was carefully transferred from the centrifugation tube into a $4 \mathrm{ml}$ quartz cuvette for UV/ VIS analysis. Absorption spectra were recorded on a Shimadzu UV-2401 PC spectrophotometer connected to a CPS-controller thermostat kept at $25.0 \pm 0.1{ }^{\circ} \mathrm{C}$.

The kinetic experiments were performed using 6-12 phosphor-coated fluorescent lamps for irradiating the samples. Rate constants for ARS degradation were obtained by monitoring the change of absorbance at the maximum wavelength $(423 \mathrm{~nm})$ as a function of the irradiation time at $25{ }^{\circ} \mathrm{C}$. The $k$ values were the average of at least three determinations. The average error estimated on the rate constants was $\pm 10 \%$.

Product analysis was carried out as follows. An aqueous dispersion of $\mathrm{TiO}_{2}(40 \mathrm{mg}$ in $100 \mathrm{ml})$ was irradiated in the presence of $1.0 \mathrm{mM}$ CTABr for $30 \mathrm{~min}$. Subsequently, the semiconductor particles were removed by centrifugation after adding of ethanol and $\mathrm{HCl}$ (see above). The supernatant was carefully transferred from the centrifugation tubes into a separating funnel and water was added to the mixture. The aqueous layer was extracted three times with chloroform. The residue, obtained after removal of the solvent under reduced pressures from the combined organic extracts, was diluted with methanol. A LCMS-ESI analysis was performed on the diluted samples by an Agilent 6540 UHD accurate mass Q-TOF LC/MSMS instrument. The analysis of the irradiated samples was always compared with that of the corresponding not-irradiated samples, prepared following the same experimental procedure. The chromatogram highlighted a main peak at 0.484 min acquisition time, which was assigned to phthalic acid by MS spectrum (negative ion acquisition): calcd for $\mathrm{C}_{8} \mathrm{H}_{5} \mathrm{O}_{4}[\mathrm{M}$ $-\mathrm{H}]^{-}$: 165.1266, found: 165.0199 (data in ESI $\dagger$ ).

The photostability of the surfactant was checked as follows. An aqueous dispersion of $\mathrm{TiO}_{2}(20 \mathrm{mg}$ in $50 \mathrm{ml})$ was irradiated in the presence of $50 \mathrm{mM} \mathrm{CTABr}$, the surfactant taken as test, for $30 \mathrm{~min}$. Subsequently, the semiconductor particles were removed by centrifugation (see above) after adding of $\mathrm{HCl}$ and the supernatant was analyzed directly. The analysis of the irradiated samples was always compared with that of the corresponding not-irradiated samples, prepared following the same experimental procedure. The photostability of the surfactant was monitored by IR spectroscopic methods, using a Perkin Elmer Spectrum RX I FT-IR System instrument. The FTIR spectra were calculated from 16 scans at $1 \mathrm{~cm}^{-1}$ resolution. The intensities of the bands at 2924 and $2854 \mathrm{~cm}^{-1}$ (C-H stretching modes) and at $1470 \mathrm{~cm}^{-1}$ (C-H bending modes), all mainly due to the methylene tails, were evaluated before and after irradiation (data in ESI $\dagger$ ). ${ }^{30}$

\section{Micellar parameter determination by surface tension measurements}

The critical micellar concentration $(\mathrm{cmc})$ values of the various cationic surfactants were measured at $\mathrm{pH} 12$ in the absence of $\mathrm{TiO}_{2}$ according to the Du Noüy ring method using a Fisher Surface Tensiometer (manual model). The measurements were performed on solutions (from 1.0 to $0.0050 \mathrm{mM}$ ) prepared by adding rates of a surfactant stock solution. The $\mathrm{cmc}$ values were obtained by the intersection of the two linear correlations obtained in the graph of the surface tensions in function of $-\log$ [surfactant], whereas the ionization degree $(\alpha)$ was determined by the ratio of the slopes of the two correlations. The average value of three measurements at $25^{\circ} \mathrm{C}$ was taken as the result. The average error was estimated to be $c a .5 \%$.

\section{Results and discussion}

\section{$\mathrm{TiO}_{2}$-photocatalyzed degradation of ARS assisted by surfactants}

In a previous paper, we reported that the $\mathrm{TiO}_{2}$-photodegradation efficiency of ARS assisted by cetyltrimethylammonium salts improved by increasing the $\mathrm{pH}$ value up to $12 .{ }^{19} \mathrm{The} \mathrm{pH}$ affected both the amount of hydroxyl radicals produced by oxidation of $\mathrm{OH}^{-}$ions available on the semiconductor surface ${ }^{7}$ and also the stabilization, due to the electrostatic attraction between the photocatalyst surface (negatively charged at this $\mathrm{pH}$ ) and the cation radical intermediates. On the basis of these results, all the experiments presented in this work were carried out at $\mathrm{pH} 12$.

Single-chain surfactants. It is known that excited Alizarin red adsorbed on the $\mathrm{TiO}_{2}$ surface was subjected to oxidation following a photo-assisted mechanism. ${ }^{31}$ ARS photodegradation produced, in the absence of additives, species as hydrogen peroxide and phthalic acid as principal products, in addition to carbon dioxide and smaller carbonyl compounds. Phthalic acid was also detected by HPLC-HRMS analysis (see Experimental) in 
our experimental condition, where the degradation of Alizarin red was performed under UV light irradiation of the semiconductor in the presence of CTABr. In this case a photocatalytic degradation mechanism should be operative, ${ }^{19}$ according to which conduction band electrons and valence band holes are formed by $\mathrm{TiO}_{2}$ irradiation in the primary step..$^{\mathbf{8 , 9}}$ Evidently, the same crucial intermediates, such as ARS radical cations and oxygen reactive species, are involved in the photodegradation process in both the irradiation conditions.

Blank experiments, performed on irradiated ARS solutions but not containing $\mathrm{TiO}_{2}$, or on dark $\mathrm{TiO}_{2} / \mathrm{ASR}$ dispersions, showed that no dye degradation occurred along a time shorter than $120 \mathrm{~min}$. It is also well known that the surfactant molecules themselves are photocatalytic degraded over $\mathrm{TiO}_{2}$ powder, ${ }^{32,33}$ but we checked by FTIR analysis (data in ESI $\dagger$ ), that the photooxidation of cetyltrialkylammonium bromide, the surfactant taken as test, by irradiated semiconductor particles is rather slow during the time in which ARS degrades. Indeed, the $\mathrm{CTABr}$ disappearance percentage, measured as transmittance change of the surfactant characteristic peaks (see Experimental) before and after the irradiation, is less than $2 \%$.

The effect of single-tailed surfactants has been examined in terms of variation of the headgroup size in the CTRABr series (Scheme 1). For this purpose, the experiments were performed in the presence of surfactants at concentrations ranging between 0.20 and $5.0 \mathrm{mM}$, keeping constant the ARS and $\mathrm{TiO}_{2}$ concentrations.

The shape of the absorption spectra recorded after $15 \mathrm{~min}$ irradiation in the presence of surfactant were very similar to those recorded without surfactant (as an example, in Fig. S1 and $\mathrm{S} 2 \dagger$ the spectra in the presence of CTABr and CTBABr are shown); this indicates that the surfactant role is mainly to affect the process kinetics. The degradation profiles, shown in Fig. 1, are reported as the changes of concentration of ARS as a function of [CTRAX]; the changes of ARS concentration are expressed as the ratio of residual ARS concentration after 15 min irradiation $\left(C_{15}\right)$ on its initial concentration $\left(C_{0}\right)$ (both values determined by absorbance measurements, see Experimental). The first observation emerging from the analysis of these profiles is that the photocatalytic efficiency depends

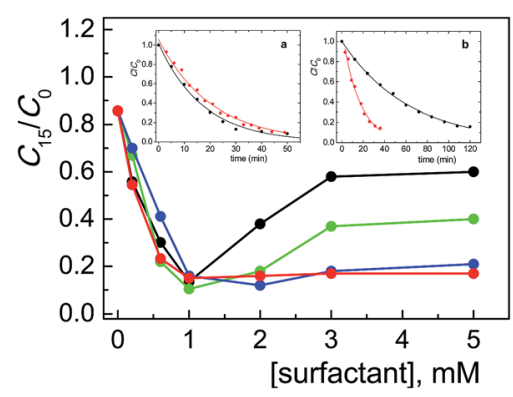

Fig. 1 Photodegradation of ARS $(0.20 \mathrm{mM})$ with $\mathrm{TiO}_{2}\left(0.4 \mathrm{~g} \mathrm{l}^{-1}\right)$ at $\mathrm{pH}$ 12 after 15 min of irradiation as a function of $\operatorname{CTABr}(\bullet), C \operatorname{CTEAr}(\bullet)$, $\mathrm{CTPABr}(\bullet)$ and $\mathrm{CTBABr}(\bullet)$ concentration. Insets: photodegradation kinetics in the presence of $\mathrm{CTABr}(\bullet)$ and $C \operatorname{TBABr}(\bullet)$ at (a) $1.0 \mathrm{mM}$ and (b) $5.0 \mathrm{mM}$ concentration. The solid lines represent the corresponding first order kinetic fittings. weakly on the surfactant structure up to a concentration of about $1 \mathrm{mM}$, where the maximum efficiency of ARS degradation is reached (about 90\%). By comparing the minimum value of the ratio $C_{15} / C_{0}(c a .0 .1)$ with that determined in the absence of CTRAX (ca. 0.9), it is possible to establish a significant catalytic efficiency by the surfactant of almost 10 times. This concentration is well above the cmc values of all CTRAX examined; indeed, the cmc values ranged from 0.23 to $0.056 \mathrm{mM}$ in alkaline medium (Table 1) increasing the chain length of the alkyl residues of the headgroup. As expected, the cmc values at $\mathrm{pH} 12$ are lower than in neutral water (Table 1), according to the effect due to the addition of ionic species (as $\mathrm{Na}^{+} \mathrm{OH}^{-}$). The second interesting evidence is that, for surfactant concentration above $1.0 \mathrm{mM}$, the photocatalytic degradation of the dye is strongly influenced on the headgroup structure. In particular, the extent of photodegradation does not change with propyl- and butyl-in the headgroup, while with methyl and ethyl it decreases rapidly with the monomer concentration until reaching a plateau value (at ca. $3 \mathrm{mM}$ surfactant), corresponding to $40 \%$ and $60 \%$ of degraded Alizarin, respectively.

These results are confirmed by kinetic analysis of the degradation obtained in the presence of CTABr or CTBABr, the two surfactants at the extremes of the degradation efficiency. Indeed, the degradation data $\left(C / C_{0} v s\right.$. time $)$, determined on 1.0 mM CTRAX solutions, were well fitted by a pseudo-first order kinetic law (inset a in Fig. 1) with slightly different observed rate constants $\left(0.050\right.$ and $0.058 \mathrm{~min}^{-1}$ for $\mathrm{CTBABr}$ and $\mathrm{CTABr}$, respectively). In the presence of $5.0 \mathrm{mM}$ surfactant the observed first order rate constant with CTBABr $\left(0.061 \mathrm{~min}^{-1}\right)$ was found similar to that at $1.0 \mathrm{mM}$ and greater than that determined with CTABr $\left(0.016 \mathrm{~min}^{-1}\right)$ by a factor of $c a .4$ (inset b in Fig. 1), which is almost the same reactivity ratio determined after 15 minutes of irradiation.

The graphics reported in Fig. 1 are very similar to that already reported for the same system, $\mathrm{ARS} / \mathrm{TiO}_{2} / \mathrm{pH} 12$, but in the presence of cetyltrimethylammonium salt with different counterions (CTAX), ${ }^{19}$ at least as regards the trend of the degradation efficiency up to $1.0 \mathrm{mM}$ surfactant concentration, which corresponds to the ARS degradation maximum. Also in this case the formation of positively charged close-packed

Table 1 Values of $\mathrm{cmc}$ and $\alpha$ in water and $\mathrm{cmc}$ in $\mathrm{NaOH} 0.01 \mathrm{M}$ at $25.0^{\circ} \mathrm{C}$

\begin{tabular}{lllll}
\hline & $\mathrm{NaOH}^{a}(\mathrm{pH} \mathrm{12})$ & & $\mathrm{H}_{2} \mathrm{O}^{b}$ \\
\cline { 2 - 2 } Surfactant & $\mathrm{cmc}(\mathrm{mM})$ & & $\mathrm{cmc}(\mathrm{mM})$ & $\alpha$ \\
\hline CTABr & 0.23 & 0.92 & 0.25 \\
$\mathrm{CTEABr}$ & 0.24 & 0.79 & 0.36 \\
CTPABr & 0.15 & 0.56 & 0.46 \\
CTBABr & 0.056 & 0.33 & 0.51 \\
DTABr & 1.5 & 1.6 & 0.28 \\
Obis(CTABr) & 0.0049 & 0.033 & 0.49 \\
Obis(DTABr) & & 1.0 & 0.26 \\
${ }^{a}$ Obtained by & surface tension & measurements. ${ }^{b}$ Determined by \\
conductivity. ${ }^{34}$ & & &
\end{tabular}


bilayers on the $\mathrm{TiO}_{2}$ surface is supposed, ${ }^{\mathbf{1 1 , 3 0}}$ which has the role of approaching the dye to the semiconductor, otherwise impossible being both negatively charged. Interestingly, in this range of surfactant concentrations, the ARS degradation efficiency is very little affected both by the steric hindrance of the headgroup alkyl chains and by the cmc values (Table 1); this probably can be due to a combination of hydrophobic and electrostatic interactions.

At monomer concentrations higher than $1.0 \mathrm{mM}$, the dye competed between two association sites, the $\mathrm{TiO}_{2}$ surface and the micellar aggregates of $\mathrm{CTABr}$ and $\mathrm{CTEABr}$, while with larger headgroup size (CTPABr and CTBABr) the degradation efficiency did not undergo any variation. In particular, the dye oxidation efficiency decreased in the order of alkyl chain length methyl < ethyl < propyl $\cong$ butyl, i.e. by decreasing the micellar dissociation degree $\alpha$ (Table 1 ), a parameter correlated with the positive charge density on the micellar surface. ${ }^{34}$ In the case of CTAX surfactants, an opposite behavior was instead observed: micelles more ionized (with greater $\alpha$ value) were able to bind more efficiently the negatively charged dye, subtracting it from the photodegradation process. The association degree of ARS to the micellar aggregates evidently does not depends only on the charge density, but also on the hydrophobic nature of the micelle surface. Previous studies showed that an increase in the headgroup size not only lowered the $\mathrm{cmc}$ value and increased the $\alpha$ parameter, but also reduced the water content and the hydrophobicity of the interface. ${ }^{27}$ In particular, the alkyl chains of CTPABr and CTBABr did not extend into the water, but were oriented along the micellar surface to reduce water-alkyl group contact. Therefore, the reduced inhibition of the photodegradation with increasing of the headgroup size can be interpreted in terms of increased difficulty of ARS to intercalate into the micelle because of steric effect due to the alkyl chains. We observed this effect also with other aromatic molecules (sodium trans-orthomethoxycinnamate) not intercalating in CTBABr micelles due to steric hindrance of the headgroups in other previous studies. ${ }^{34}$

Double-tailed surfactants. Two dimeric quaternary ammonium surfactants with $C_{16}$ and $C_{12}$ tail length and same diethylene oxide spacer (ObisCTABr and ObisDTABr) were compared with the corresponding monomeric surfactants (CTABr and DTABr). Based on previous studies on aggregate structures at solid-liquid interface as a function of surfactant geometry, ${ }^{35,36}$ it is plausible to assume that ObisCTABr and ObisDTABr, being symmetric geminis and with large spacer, show spherical micelle geometry in neat water and hemimicellar/admicelle structures adsorbed on the negatively charged $\mathrm{TiO}_{2}$ surface, similarly to what is observed with the correspondent singletailed surfactants.

In Fig. 2 the degradation profiles of the concentration change of ARS, expressed as $C_{15} / C_{0}$ ratio as a function of surfactant concentration, for ObisCTABr and CTABr are compared. Two most significant aspects emerged from the analysis of these profiles. The first one was that the maximum photocatalytic efficiency for ObisCTABr, ca. 65\% of degraded ARS, was at a concentration (0.2 mM) 5 times lower than that for $\mathrm{CTABr}(1 \mathrm{mM})$. Both values are higher than the corresponding

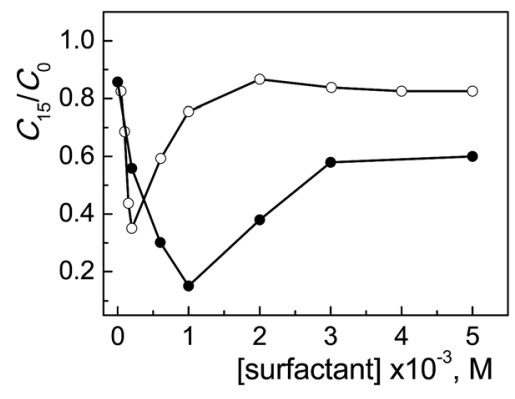

Fig. 2 Photodegradation of ARS $(0.20 \mathrm{mM})$ with $\mathrm{TiO}_{2}\left(0.4 \mathrm{~g} \mathrm{l}^{-1}\right)$ at $\mathrm{pH}$ 12 after 15 min of irradiation as a function of $\mathrm{ObisCTABr}(\mathrm{O})$ and $\mathrm{CTABr}$ (O) concentration.

cmc values ( 0.049 and $2.3 \mathrm{mM}$ at $\mathrm{pH} 12$, Table 1$)$. The other relevant aspect concerns the shape of the profiles, namely the curve appeared less broaden going from CTABr to ObisCTABr, reaching a plateau value from $c a$. $60 \%$ to $82 \%$ of residual ARS.

The highest inhibition effect on the dye degradation process observed with ObisCTABr is in line with its much lower cmc value and higher $\alpha$ parameter (cmc: 0.049 and $0.23 \mathrm{mM}, \alpha: 0.36$ and 0.25 for ObisCTABr and $\mathrm{CTABr}$, respectively, Table 1). Indeed, the lower cmc value implied that, in the presence of the same amount of surfactant, the gemini system provided a greater number of micellar aggregates able to associate ARS and thus to subtract it from the $\mathrm{TiO}_{2}$ catalytic action. Furthermore, the ObisCTABr micelle aggregates, being more ionized because less associated with the counterions, interacted more effectively with the negatively charged ARS.

The comparison of the profiles $C_{15} / C_{0}$ against the surfactant concentration obtained with ObisDTABr and DTABr (Fig. S3 in ESI $\dagger$ ) pointed out smaller differences between the two systems. The maximum efficiency of degradation with ObisDTABr ( $c a$. $85 \%$ ) was obtained at $0.5 \mathrm{mM}$, a concentration that is half of the value found for the corresponding single-tailed DTABr (maximum efficiency ca. $80 \%$ ); the $C_{15} / C_{0}$ values at the plateau in this case were more similar $(0.7$ and 0.63 for ObisDTABr and $\mathrm{DTABr}$, respectively). These results were in line with the similar cmc and $\alpha$ values for the two systems, as observed from the data collected in Table 1 (cmc: 1.0 and 1.6, $\alpha$ : 0.26 and 0.28 for ObisDTABr and DTABr, respectively). In this case the favorable adsorption by electrostatic and hydrophobic interaction of ObisRTABr surfactants on the $\mathrm{TiO}_{2}$, due to the double charge and double chain in its structure, seemed to play a role only in anticipating, in terms of concentration, the maximum photodegradation point of ARS.

\section{$\mathrm{TiO}_{2}$-photocatalyzed degradation of ARS assisted by tetraalkyl quaternary ammonium salts}

In order to investigate the role of the amphiphilic character of the quaternary ammonium salts in assisting the $\mathrm{TiO}_{2}$ photocatalyzed degradation of Alizarin, we examined numerous bromide salts, with variously structured ammonium groups, not able to form micelles in the same experimental conditions described above. 
Effect on the chain length of the alkyl groups. In Fig. $3 C_{15} / C_{0}$ ratios as a function of salt concentration profiles of tetramethyl, tetraethyl-, tetrapropyl- and tetrabutylammonium bromide (TMABr, TEABr, TPABr and TBABr, respectively, Scheme 2), are collected. With all the ammonium salts, the efficiency degradation of ARS increased with increasing both the salt concentration and, at the same salt concentration, the length of the alkyl chains. At concentrations $\geq 50 \mathrm{mM}$ the catalytic effect reached a constant maximum value for all the investigated systems.

Comparing these results with those collected with the corresponding surfactants (CTABr, CTEABr, CTPABr and CTBABr), the main evidences were: (i) the maximum catalytic effect was still very high, of the same entity (90\%) but reached at much higher concentration of salt; (ii) the inhibition effect due to the competition with the micelles was obviously missing; (iii) the chain length effect of the alkyl residue on the efficiency of the degradation process was roughly kept, i.e., butyl $>$ propyl $>$ ethyl $>$ methyl. Indeed tetrabutylammonium, being the softest cation, neutralized better the negative charge of the dye, which is also soft since the charge is delocalized on the aromatic rings. This neutralization could allow a more effective approach of the dye to the semiconductor, a necessary condition to facilitate kinetically the electron transfer from the dye to the positive hole photogenerated on the $\mathrm{TiO}_{2}$ surface. At the same time, the tetrabutylammonium group could create a coating on the catalyst surface not only by electrostatic interactions, but also by hydrophobic interactions between the butyl chains, reducing in this way their steric hindrance. The trimethylammonium, being the hardest of the four salts examined, could prefer to interact electrostatically by the positive charged nitrogen with the surface hard $\mathrm{TiO}^{-}$groups, rather than with the dye. ${ }^{7}$ So, the less Alizarin molecules were in proximity of the semiconductor, the less efficient was their photodegradation. TEABr and TPABr clearly followed the trend due to the hydrophobicity of the system.

The concentration of $50 \mathrm{mM}$, in correspondence of which the photodegradation reached its maximum value, corresponds to the situation of maximum adsorbed amount of Alizarin on the semiconductor in the presence of different salts. It is plausible that the formation of positive charged close-packed bilayers of surfactant molecules adsorbed on the $\mathrm{TiO}_{2}$ surface, which are

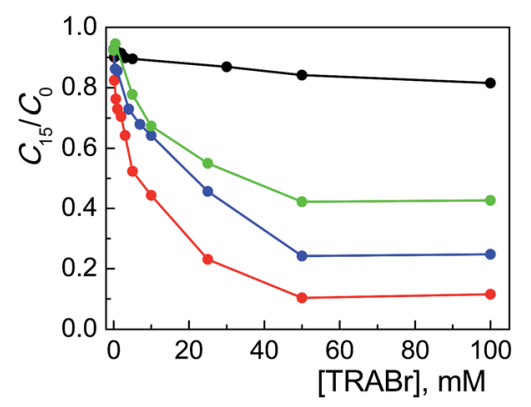

Fig. 3 Photodegradation of ARS $(0.20 \mathrm{mM})$ with $\mathrm{TiO}_{2}\left(0.4 \mathrm{~g} \mathrm{l}^{-1}\right)$ at $\mathrm{pH}$ 12 after 15 min of irradiation as a function of $\operatorname{TMABr}(\bullet), \operatorname{TEABr}(\bullet)$, $\operatorname{TPABr}(\bullet)$ and $\operatorname{TBABr}(\bullet)$ concentration. known to assist the ARS degradation in micellar media, ${ }^{\mathbf{1 9}}$ was achieved at lower surfactant concentrations because of the importance of non-electrostatic hydrocarbon tail-tail interactions.

Effect of the conformational freedom. To deepen the intervention of structural change of the salt on the ARS oxidative degradation, the influence of the steric hindrance of the groups on the charged ammonium was examined. For this purpose, the derivatives of aza-spiro bromide (ASBr) quinuclidinium bromide (BQBr) and pyridinium bromide (BPyBr), together with the acyclic salt TPABr were investigated in the same experimental condition already reported. The corresponding structures are depicted in Scheme 2.

From the $C_{15} / C_{0}$ ratio as a function of salt concentration profiles shown in Fig. 4, it is observable that the catalytic efficiency decreased going from the structure with a higher degree of conformational freedom, such that of TPABr, to the more rigid molecular structures of $\mathrm{BQBr}$ and $\mathrm{ASBr}$. To explain this trend, the hydrophobicity of alkyl residues can not be invoked, since the number of the carbon atoms is similar. Indeed, in the case of $\mathrm{BQBr}$ and $\mathrm{ASBr}$, the ammonium positive charge should have been more available for the interaction both with the dye and with the negatively charged catalyst surface, compared with the higher steric hindrance of TPABr.

The analogous behavior of $\mathrm{BQBr}$ and $\mathrm{ASBr}$ suggests that the ammonium charge availability is similar with the two salts. Evidently, in the BQBr structure, the conformational freedom of the butyl residue is balanced by the rigidity of quinuclidinium portion to the same extent of the two rigid chair conformation of ASBr.

The weaker effect of BPyBr on the degradation reaction may seem unexpected. Also in this case the hydrophobicity can not be influent, since its carbon atom number similar to that of the others $(9,12,11$ and 10 for $\mathrm{BPyBr}, \mathrm{TPABr}, \mathrm{BQBr}$ and $\mathrm{ASBr}$, respectively). The rigidity of the $\mathrm{BPyBr}$ should have determined a better accessibility of the ammonium, but two main factors could hinder the neutralization: the first is electronic cloud of the aromatic ring that repulses the negatively charged dye and then the approach of the dye to the semiconductor; the second factor could be the different hybridization of the nitrogen atom $\left(\mathrm{sp}^{2}\right.$ in pyridinium, $\mathrm{sp}^{3}$ in the other cases) that determines a lower positive charge density on nitrogen.

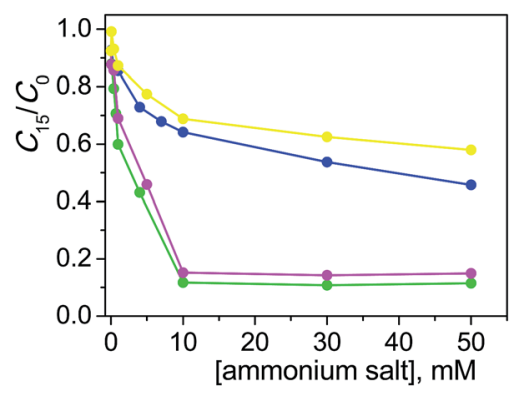

Fig. 4 Photodegradation of ARS $(0.20 \mathrm{mM})$ with $\mathrm{TiO}_{2}\left(0.4 \mathrm{~g} \mathrm{l}^{-1}\right)$ at $\mathrm{pH}$ 12 after 15 min of irradiation as a function of $\operatorname{TPABr}(\bullet), \operatorname{BPyBr}(\circ), \mathrm{ASBr}$ (๑), $\operatorname{BQBr}(\bullet)$. 


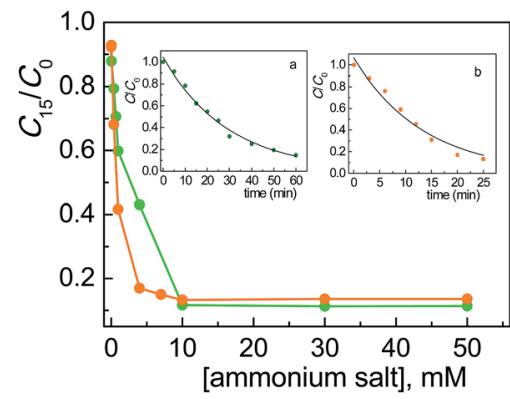

Fig. 5 Photodegradation of ARS $(0.20 \mathrm{mM})$ with $\mathrm{TiO}_{2}\left(0.4 \mathrm{~g} \mathrm{l}^{-1}\right)$ at $\mathrm{pH}$ 12 after $15 \mathrm{~min}$ of irradiation as a function of $\mathrm{ASBr}(\bullet), \operatorname{DASBr}(\bullet)$ concentrations. Insets: photodegradation kinetics in the presence of $1.0 \mathrm{mM} \mathrm{ASBr}$ (a) and $\mathrm{DASBr}$ (b) and corresponding kinetic fittings.

Effect of the number of positive charges. The effect of the charge number of the salt on the photodegradation efficiency was evaluated considering the two aza spiro systems ASBr and DASBr (Scheme 2). As observed in Fig. 5, where the $C_{15} / C_{0} v s$. concentration profiles are shown, with both the salts an excellent percentage degradation ( $c a$. 90\%) was obtained at high concentrations. The interesting difference, instead, was that with ASBr the degradation process was slower; indeed the dye degradation maximum was reached at $10 \mathrm{mM}$ concentration, a doubled value than that observed with DASBr $(5 \mathrm{mM})$. This was confirmed by kinetic analysis performed with the two salts (both $1.0 \mathrm{mM}$ ). The observed first order rate constant of the degradation process assisted by $\operatorname{ASBr}\left(0.034 \mathrm{~min}^{-1}\right)$ was found smaller than that determined with DASBr $\left(0.071 \mathrm{~min}^{-1}\right)$ by a factor of $c a$. 0.5. These results indicated that the influence of the two salts could be ascribed to a pure charge concentration effect of charge concentration on the oxidative process kinetic.

\section{Conclusions}

The addition of CTRABr surfactants significantly enhanced ARS decomposition kinetics on the $\mathrm{TiO}_{2}$ surface in air-equilibrated alkaline aqueous dispersions under UV light irradiation. This effect could be mainly explained by the increased approach of ARS molecules to the solid surface of the semiconductor through hemimicelle/admicelle structures adsorbed on the $\mathrm{TiO}_{2}$ particles. This facilitated the reaction of the dye with the photo-yielded oxidative species formed on the semiconductor surface. The ARS photodegradation rate reached a maximum value when the surfactant concentration is about $1 \mathrm{mM}$, a value lower than the cmc of all examined surfactants. Above this value, the ARS degradation rate is reduced because of the competition of the micellar aggregates in adsorbing the dye. These results were also compared with that obtained with analogous surfactant in which structure length and number of the tails have been varied.

The assistance of non-amphiphilic quaternary ammonium salts to the $\mathrm{TiO}_{2}$ catalyzed photodegradation of ARS was realized by reducing the electrostatic repulsion between the anionic dye and the negatively charged semiconductor. The results collected in this study highlighted that the salt action is accomplished by its hydrophobic character, the availability of the positive charge on the ammonium group and the number of charged nitrogen atoms.

The advantages of using non-amphiphilic salts were that the dye photodegradation process did not undergo inhibition, although the lack of hydrophobic tails required greater salt concentrations to achieve values of degradation efficiency comparable to those obtained with the surfactants. This effect was however offset by lower environmental impact of nonamphiphilic alkylammonium salts with respect to the corresponding surfactants.

\section{Acknowledgements}

Thanks are due to MIUR (Ministero dell'Istruzione, dell'Università e della Ricerca Scientifica), Italy (PRIN 20102011, grant number 2010FM738P) and to the University of Perugia (Fondo Ricerca di Base 2015) for financial support.

\section{References}

1 P. B. S. Ratna, Int. J. Environ. Sci., 2012, 3, 940-955.

2 B. R. Babu, A. Parande, S. Raghu and T. P. Kumar, J. Cotton Sci., 2007, 11, 141-153.

3 O. J. Hao, H. Kim and P.-C. Chiang, Crit. Rev. Environ. Sci. Technol., 2000, 30, 449-505.

4 U. Wiesmann, I. S. Choi and E.-M. Dombrowski, Fundamentals of biological wastewater treatment, Wiley-VCH Verlag GmbH \& Co. KGaA, 2007.

5 Y. Anjaneyulu, N. S. Chary and D. S. S. Raj, Rev. Environ. Sci. Bio/Technol., 2005, 4, 245-273.

6 C. Zaharia, D. Suteu, A. Muresan, R. Muresan and A. Popescu, Environ. Eng. Manage. J., 2009, 8, 1359-1369.

7 U. Akpan and B. Hameed, J. Hazard. Mater., 2009, 170, 520529.

8 G. A. Epling and C. Lin, Chemosphere, 2002, 46, 561-570.

9 I. K. Konstantinou and T. A. Albanis, Appl. Catal., B, 2004, 49, 1-14.

10 T. Tachikawa, M. Fujitsuka and T. Majima, J. Phys. Chem. C, 2007, 111, 5259-5275.

11 R. Zhang and P. Somasundaran, Adv. Colloid Interface Sci., 2006, 123, 213-229.

12 T. H. Talib, M. A. AlDamen and R. R. Alani, Am. Chem. Sci. J., 2014, 4, 918-933.

13 K. Joshi and V. Shrivastava, Int. J. Environ. Sci., 2011, 2, 8-21. 14 A. Houas, H. Lachheb, M. Ksibi, E. Elaloui, C. Guillard and J.-M. Herrmann, Appl. Catal., B, 2001, 31, 145-157.

15 M. L. de Souza and P. Corio, Appl. Catal., B, 2013, 136, 325333.

16 M. Bettoni, T. Del Giacco, C. Rol and G. V. Sebastiani, J. Photochem. Photobiol., A, 2007, 190, 34-40.

17 M. Bettoni, T. Del Giacco, F. Elisei, C. Rol and G. V. Sebastiani, Phys. Chem. Chem. Phys., 2010, 12, 54255430 .

18 M. Bettoni, L. Brinchi, T. Del Giacco, R. Germani, S. Meniconi, C. Rol and G. V. Sebastiani, J. Photochem. Photobiol., A, 2012, 229, 53-59. 
19 T. Del Giacco, R. Germani, F. Saracino and M. Stradiotto, J. Photochem. Photobiol., A, 2017, 332, 546-553.

20 M. Tiecco, L. Roscini, L. Corte, C. Colabella, R. Germani and G. Cardinali, Langmuir, 2016, 32, 1101-1110.

21 L. Corte, M. Tiecco, L. Roscini, R. Germani and G. Cardinali, Colloids Surf., B, 2014, 116, 761-771.

22 M. Tiecco, P. Di Profio, R. Germani and G. Savelli, Nucleosides, Nucleotides Nucleic Acids, 2009, 28, 911-923.

23 M. J. Rosen and J. T. Kunjappu, Surfactants and interfacial phenomena, John Wiley \& Sons, 2012.

24 Y. Zhang, H. Wu, J. Zhang, H. Wang and W. Lu, J. Hazard. Mater., 2012, 221, 92-99.

25 D. Fabbri, A. Crime, M. Davezza, C. Medana, C. Baiocchi, A. B. Prevot and E. Pramauro, Appl. Catal., B, 2009, 92, 318-325.

26 M. Davezza, D. Fabbri, E. Pramauro and A. B. Prevot, Environ. Sci. Pollut. Res., 2013, 20, 3224-3231.

27 R. Germani, P. P. Ponti, T. Romeo, G. Savelli, N. Spreti, G. Cerichelli, L. Luchetti, G. Mancini and C. A. Bunton, J. Phys. Org. Chem., 1989, 2, 553-558.
28 R. A. Aitken, E. F. Philp, F. G. Riddell and M. H. Smith, ARKIVOC, 2002, 3, 63-70.

29 T. Tachikawa, S. Tojo, M. Fujitsuka and T. Majima, Chem.Eur. J., 2006, 12, 3124-3131.

$30 \mathrm{H}$. Li and C. P. Tripp, Langmuir, 2002, 18, 9441-9446.

31 J. Zhao, T. Wu, K. Wu, K. Oikawa, H. Hidaka and N. Serpone, Environ. Sci. Technol., 1998, 32, 2394-2400.

32 B. Singhal, A. Porwal, A. Sharma, R. Ameta and S. C. Ameta, J. Photochem. Photobiol., A, 1997, 108, 85-88.

33 R. Zhang, L. Gao and Q. Zhang, Chemosphere, 2004, 54, 405411.

34 P. Baglioni, E. Braccalenti, E. Carretti, R. Germani, L. Goracci, G. Savelli and M. Tiecco, Langmuir, 2009, 25, 5467-5475.

35 S. Manne, T. Schäffer, Q. Huo, P. Hansma, D. Morse, G. Stucky and I. Aksay, Langmuir, 1997, 13, 6382-6387.

36 S. Ardizzone, C. Bianchi, P. Quagliotto and G. Viscardi, Surf. Interface Anal., 2002, 34, 652-656. 